\title{
Interacting generalized Chaplygin gas model in non-flat universe
}

\author{
M.R. Setare * \\ Department of Science, Payame Noor University. Bijar, Iran
}

\begin{abstract}
We employ the generalized Chaplygin gas of interacting dark energy to obtain the equation of state for the generalized Chaplygin gas energy density in non-flat universe. By choosing a negative value for $B$ we see that $w_{\Lambda}^{\text {eff }}<-1$, that corresponds to a universe dominated by phantom dark energy.
\end{abstract}

${ }^{*}$ E-mail: rezakord@ipm.ir 


\section{Introduction}

One of the most important problems of cosmology, is the problem of so-called dark energy (DE). The type Ia supernova observations suggests that the universe is dominated by dark energy with negative pressure which provides the dynamical mechanism of the accelerating expansion of the universe $[1,2,3]$. The strength of this acceleration is presently matter of debate, mainly because it depends on the theoretical model implied when interpreting the data. Most of these models are based on dynamics of a scalar or multi-scalar fields. Primary scalar field candidate for dark energy was quintessence scenario[4,5], a fluid with the parameter of the equation of state lying in the range, $-1<w<\frac{-1}{3}$. While the most model independent analysis suggest that the acceleration of the universe to be below the de Sitter value [6], it is certainly true that the body of observational data allows for a wide parameter space compatible with an acceleration larger than the de Sitter's [7, 8]. If eventually this proves to be the case, the fluid driving the expansion would violate not only the strong energy condition $\rho+3 P>0$, but the dominate energy condition $\rho+P>0$, as well. Fluids of such characteristic dubbed phantom fluid [9]. In spite of the fact that the field theory of phantom fields encounter the problem of stability which one could try to bypass by assuming them to be effective fields $[10,11]$, it is nevertheless interesting to study their cosmological implication. Recently there are many relevant studies on phantom energy [12]. The analysis of the properties of dark energy from recent observations mildly favor models with $w$ crossing -1 in the near past. So far, a large class of scalar-field dark energy models have been studied, including quintessence, K-essence [13], tachyon [14], ghost condensate [15, 16] and quintom [17], and so forth. In addition, other proposals on dark energy include interacting dark energy models [18], braneworld models [19], and holographic dark energy models [20], etc..

In a very interesting paper Kamenshchik, Moschella, and Pasquier [21]have studied a homogeneous model based on a single fluid obeying the Chaplygin gas equation of state

$$
P=\frac{-A}{\rho}
$$

where $P$ and $\rho$ are respectively pressure and energy density in comoving reference frame, with $\rho>0 ; A$ is a positive constant. This equation of state has raised a certain interest [22] because of its many interesting and, in some sense, intriguingly unique features. Some possible motivations for this model from the field theory points of view are investigated in [23]. The Chaplygin gas emerges as an effective fluid associated with d-branes [24] and can also be obtained from the Born-Infeld action [25].

Inserting the equation of state (1) into the relativistic energy conservation equation, leads to a density evolving as

$$
\rho_{\Lambda}=\sqrt{A+\frac{B}{a^{6}}}
$$

where $B$ is an integration constant.

In present paper, using the generalized Chaplygin gas model of dark energy, we obtain equation of state for interacting Chaplygin gas energy density in non-flat universe. The current available observational data imply that the dark energy behaves as phantom-type dark energy, i.e. the equation-of-state of dark energy crosses the cosmological-constant boundary $w=-1$ during the evolution history. We show this phantomic description of the interacting generalized Chaplygin gas dark energy in non-flat universe with $B<0$. 


\section{Interacting generalized Chaplygin gas}

In this section we obtain the equation of state for the generalized Chaplygin gas when there is an interaction between generalized Chaplygin gas energy density $\rho_{\Lambda}$ and a Cold Dark Matter(CDM) with $w_{m}=0$. The continuity equations for dark energy and CDM are

$$
\begin{aligned}
& \dot{\rho}_{\Lambda}+3 H\left(1+w_{\Lambda}\right) \rho_{\Lambda}=-Q, \\
& \dot{\rho}_{\mathrm{m}}+3 H \rho_{\mathrm{m}}=Q .
\end{aligned}
$$

The interaction is given by the quantity $Q=\Gamma \rho_{\Lambda}$. This is a decaying of the generalized Chaplygin gas component into CDM with the decay rate $\Gamma$. Taking a ratio of two energy densities as $r=\rho_{\mathrm{m}} / \rho_{\Lambda}$, the above equations lead to

$$
\dot{r}=3 H r\left[w_{\Lambda}+\frac{1+r}{r} \frac{\Gamma}{3 H}\right]
$$

Following Ref.[26], if we define

$$
w_{\Lambda}^{\mathrm{eff}}=w_{\Lambda}+\frac{\Gamma}{3 H}, \quad w_{m}^{\mathrm{eff}}=-\frac{1}{r} \frac{\Gamma}{3 H} .
$$

Then, the continuity equations can be written in their standard form

$$
\begin{aligned}
& \dot{\rho}_{\Lambda}+3 H\left(1+w_{\Lambda}^{\text {eff }}\right) \rho_{\Lambda}=0, \\
& \dot{\rho}_{m}+3 H\left(1+w_{m}^{\text {eff }}\right) \rho_{m}=0
\end{aligned}
$$

We consider the non-flat Friedmann-Robertson-Walker universe with line element

$$
d s^{2}=-d t^{2}+a^{2}(t)\left(\frac{d r^{2}}{1-k r^{2}}+r^{2} d \Omega^{2}\right)
$$

where $k$ denotes the curvature of space $\mathrm{k}=0,1,-1$ for flat, closed and open universe respectively. A closed universe with a small positive curvature $\left(\Omega_{k} \sim 0.01\right)$ is compatible with observations $[27,28]$. We use the Friedmann equation to relate the curvature of the universe to the energy density. The first Friedmann equation is given by

$$
H^{2}+\frac{k}{a^{2}}=\frac{1}{3 M_{p}^{2}}\left[\rho_{\Lambda}+\rho_{\mathrm{m}}\right]
$$

Define as usual

$$
\Omega_{\mathrm{m}}=\frac{\rho_{m}}{\rho_{c r}}=\frac{\rho_{\mathrm{m}}}{3 M_{p}^{2} H^{2}}, \quad \Omega_{\Lambda}=\frac{\rho_{\Lambda}}{\rho_{c r}}=\frac{\rho_{\Lambda}}{3 M_{p}^{2} H^{2}}, \quad \Omega_{k}=\frac{k}{a^{2} H^{2}}
$$

Now we can rewrite the first Friedmann equation as

$$
\Omega_{\mathrm{m}}+\Omega_{\Lambda}=1+\Omega_{k}
$$

Using Eqs. $(11,12)$ we obtain following relation for ratio of energy densities $r$ as

$$
r=\frac{1+\Omega_{k}-\Omega_{\Lambda}}{\Omega_{\Lambda}}
$$


In the generalized Chaplygin gas approach [25], the equation of state to (1) is generalized to

$$
P_{\Lambda}=\frac{-A}{\rho_{\Lambda}^{\alpha}}
$$

The above equation of state leads to a density evolution as

$$
\rho_{\Lambda}=\left[A+\frac{B}{a^{3(1+\alpha)}}\right]^{\frac{1}{1+\alpha}}
$$

Taking derivative in both sides of above equation with respect to cosmic time, we obtain

$$
\dot{\rho_{\Lambda}}=-3 B H a^{-3(1+\alpha)}\left[A+B a^{-3(1+\alpha)}\right]^{\frac{-\alpha}{1+\alpha}}
$$

Substituting this relation into Eq.(3) and using definition $Q=\Gamma \rho_{\Lambda}$, we obtain

$$
w_{\Lambda}=\frac{B}{a^{3(1+\alpha)}\left[A+B a^{-3(1+\alpha)}\right]}-\frac{\Gamma}{3 H}-1 .
$$

Here as in Ref.[29], we choose the following relation for decay rate

$$
\Gamma=3 b^{2}(1+r) H
$$

with the coupling constant $b^{2}$. Using Eq.(13), the above decay rate take following form

$$
\Gamma=3 b^{2} H \frac{\left(1+\Omega_{k}\right)}{\Omega_{\Lambda}}
$$

Substituting this relation into Eq.(17), one finds the generalized Chaplygin gas energy equation of state

$$
w_{\Lambda}=\frac{B}{a^{3(1+\alpha)}\left[A+B a^{-3(1+\alpha)}\right]}-\frac{b^{2}\left(1+\Omega_{k}\right)}{\Omega_{\Lambda}}-1 .
$$

Now using the definition generalized Chaplygin gas energy density $\rho_{\Lambda}$, and using $\Omega_{\Lambda}$, we can rewrite the above equation as

$$
w_{\Lambda}=\frac{B}{\left(3 M_{p}^{2} H^{2} a^{3} \Omega_{\Lambda}\right)^{1+\alpha}}-\frac{b^{2}\left(1+\Omega_{k}\right)}{\Omega_{\Lambda}}-1 .
$$

From Eqs.(6, 19, 21), we have the effective equation of state as

$$
w_{\Lambda}^{e f f}=\frac{B}{\left(3 M_{p}^{2} H^{2} a^{3} \Omega_{\Lambda}\right)^{1+\alpha}}-1 .
$$

By choosing a negative value for $B$ we see that $w_{\Lambda}^{\text {eff }}<-1$, that corresponds to a universe dominated by phantom dark energy. Eq.(22), for $\alpha=1$, is the effective parameter of state for Chaplygin gas. In this case, in the expression for energy density (2), term under square root should be positive, i.e. $a^{6}>\frac{-B}{A}$, then the minimal value of the scale factor is given by $a_{\min }=\left(\frac{-B}{A}\right)^{\frac{1}{6}}$, therefore according to this model we have a bouncing universe. Generally for this model $A>0, B<0$ and $1+\alpha>0$. From Eq. (15), we can realize 
that the cosmic scale factor takes values in the interval $a_{\text {min }}<a<\infty$ which corresponds to $0<\rho<(2 A)^{\frac{1}{1+\alpha}}$, where

$$
a_{\min }=\left(\frac{-B}{A}\right)^{\frac{1}{3(1+\alpha)}}
$$

Using Eq.(2), one can see that the Chaplygin gas model interpolates between dust at small $a$ and a cosmological constant at large $a$, but choosing a negative value for $B$, this quartessence idea lose. Following [21] if we consider a homogeneous scalar filed $\phi(t)$ and a potential $V(\phi)$ to describe the Chaplygin cosmology, we find

$$
\dot{\phi}^{2}=\frac{B}{a^{6} \sqrt{A+\frac{B}{a^{6}}}}
$$

Now, by choosing a negative value for $B$ we see that $\dot{\phi}^{2}<0$, then we can write

$$
\phi=i \psi
$$

In this case the Lagrangian of scalar field $\phi(t)$ can rewritten as

$$
L=\frac{1}{2} \dot{\phi}^{2}-V(\phi)=-\frac{1}{2} \dot{\psi}^{2}-V(i \psi)
$$

The energy density and the pressure corresponding to the scalar field $\psi$ are as following respectively

$$
\begin{aligned}
& \rho_{\psi}=-\frac{1}{2} \dot{\psi}^{2}+V(i \psi) \\
& P_{\psi}=-\frac{1}{2} \dot{\psi}^{2}-V(i \psi)
\end{aligned}
$$

therefore, the scalar field $\psi$ is a phantom field. This implies that one can generate phantom-like equation of state from an interacting generalized Chaplygin gas dark energy model in non-flat universe.

\section{Conclusions}

In order to solve cosmological problems and because the lack of our knowledge, for instance to determine what could be the best candidate for DE to explain the accelerated expansion of universe, the cosmologists try to approach to best results as precise as they can by considering all the possibilities they have. Within the different candidates to play the role of the dark energy, the Chaplygin gas, has emerged as a possible unification of dark matter and dark energy, since its cosmological evolution is similar to an initial dust like matter and a cosmological constant for late times. Inspired by the fact that the Chaplygin gas possesses a negative pressure, people [30] have undertaken the simple task of studying a FRW cosmology of a universe filled with this type of fluid.

In this paper, by considering an interaction between generalized Chaplygin gas energy density and CDM, we have obtained the equation of state for the interacting generalized Chaplygin gas energy density in the non-flat universe. Then we have shown that the interacting generalized Chaplygin gas dark energy with $B<0$ can be described by the phantom field. Previously I have shown that the phantom dark energy model can behave 
as a holographic dark energy [31]. In the other hand recently Zimdhal has shown that a Chaplygin gas, can be seen as special realization of a holographic dark energy cosmology if the option of an interaction between pressureless dark matter and dark energy is taken seriously [32]. In fact pressureless dark matter in interaction with holographic dark energy is more than just another model to describe an accelerated expansion of the universe. This clarify the role of interaction in this model.

\section{References}

[1] S. Perlmutter et al, Astrophys. J.517, 565, (1999).

[2] P. M. Garnavich et al, Astrophys. J, 493, L53, (1998).

[3] A. G. Riess et al, Astron. J. 116, 1009, (1998).

[4] B. Ratra and P. J. E. Peebles, Phys. Rev. D37 (1988) 3406; C.Wetterich, Nucl. Phys. B302, 302, (1988).

[5] I. Zlatev, L. Wang and P. J. Steinhardt, Phys. Rev. Lett, 82 (1999) 896; P. J. Steinhardt, L. Wang and I. Zlatev, Phys. Rev. D59 (1999) 123504.

[6] R. A. Daly and S. G. Djorgovsky, Astrophys. J. 597, 9 (2003); M. 0V. John, Astrophys. J. 614, 1 (2004); S. Nesserisand L. Perivolaroupolos, Phys. Rev. D 70, 043531 (2004); Y. Wang and M. Tegmark, Phys. Rev. D 71, 103513 (2005).

[7] R. R. Caldwell, M. Kamionkowski, and N. N. Weinberg, Phys. Rev. Lett. 91, 071301 (2003).

[8] S. Hannestad and E. Mörtsell, JCAP 04(2004) 001; Jun-Qing Xia, Gong-Bo Zhao, Bo Feng, Hong Li, Xinmin Zhang, Phys. Rev. D73, 063521, (2006).

[9] R. R. Caldwell, Phys. Lett. B 545, 23 (2002).

[10] S. M. Carroll, M. Hoffman and M. Trodden, Phys. Rev. D68 (2003) 023509.

[11] G. W. Gibbons, hep-th/0302199; A. E. Schulz and M. White, Phys. Rev. D64 (2001) 043514; R. R. Caldwell, M. Kamionkowski and N. N. Weinberg, Phys. Rev. Lett. 91 (2003) 071301; M. P. Dabrowski, T. Stachowiak and M. Szydlowski, Phys. Rev. D68 (2003) 103519; P. Singh, M. Sami and N. Dadhich, Phys. Rev. D68 (2003) 023522; S. Nojiri and S. D. Odintsov, Phys. Lett. B562 (2003) 147; S. Nojiri and S. D. Odintsov, Phys. Lett. B565 (2003) 1; S. Nojiri, S. D. Odintsov, O. G. Gorbunova, J. Phys. A39, 6627, (2006); Z. K. Guo, Y. S. Piao and Y. Z. Zhang, Phys. Lett. B594 (2004) 247.

[12] X. H. Meng and P. Wang, hep-ph/0311070; V. B. Johri, astro-ph/0311293; M. Sami and A. Toporensky, Mod. Phys. Lett. A19, 1509, (2004); M. Szydlowski, W. Czaja and A. Krawiec, Phys. Rev. E72, 036221, (2005); J. Lima and J. S. Alcaniz, Phys. Lett. B 600, 191, (2004); M. Bouhmadi-Lopez and J. J. Madrid, JCAP 0505, 005, (2005); L. P. Chimento and R. Lazkoz, Int. J.Mod. Phys. D 14, 587, (2005); Y. H. Wei and Y. Tian, Class. Quant. Grav. 21, 5347, (2004); V. K. Onemli and R. P. 
Woodard, Phys. Rev. D 70, 107301, (2004); P. F. Gonzalez-Diaz and C. L. Siguenza, Nucl. Phys. B 697, 363, (2004); P. F. Gonzalez-Diaz, TSPU Vestnik 44N7, 36, (2004); Y. H. Wei, Mod. Phys. Lett. A20, 1147, (2005); S. Nojiri and S. D. Odintsov, Phys. Rev. D72, 023003, (2005); S. Capozziello, S. Nojiri and S. D. Odintsov, Phys. Lett. B632, 597, (2006); S. Nojiri and S. D. Odintsov, Gen. Rel. Grav. 38, 1285, (2006).

[13] C. Armendariz-Picon, V. F. Mukhanov and P. J. Steinhardt, Phys. Rev. Lett. 85, 4438 (2000) [astro-ph/0004134];

C. Armendariz-Picon, V. F. Mukhanov and P. J. Steinhardt, Phys. Rev. D 63, 103510 (2001) [astro-ph/0006373].

[14] A. Sen, JHEP 0207, 065 (2002) [hep-th/0203265];

T. Padmanabhan, Phys. Rev. D 66, 021301 (2002) [hep-th/0204150].

[15] N. Arkani-Hamed, H. C. Cheng, M. A. Luty and S. Mukohyama, JHEP 0405, 074 (2004) [hep-th/0312099].

[16] F. Piazza and S. Tsujikawa, JCAP 0407, 004 (2004) [hep-th/0405054].

[17] B. Feng, X. L. Wang and X. M. Zhang, Phys. Lett. B 607, 35 (2005) [astro$\mathrm{ph} / 0404224]$

Z. K. Guo, Y. S. Piao, X. M. Zhang and Y. Z. Zhang, Phys. Lett. B 608, 177 (2005) [astro-ph/0410654];

X. Zhang, Commun. Theor. Phys. 44, 762 (2005);

A. Anisimov, E. Babichev and A. Vikman, JCAP 0506, 006 (2005) [astroph/0504560];

M. R. Setare, Phys. Lett. B 641, 130, (2006);

E. Elizalde, S. Nojiri, and S. D. Odintsov, Phys. Rev. D70, 043539, (2004);

S. Nojiri, S. D. Odintsov, and S. Tsujikawa, Phys. Rev. D71, 063004, (2005).

[18] L. Amendola, Phys. Rev. D 62, 043511 (2000) [astro-ph/9908023];

D. Comelli, M. Pietroni and A. Riotto, Phys. Lett. B 571, 115 (2003) [hep$\mathrm{ph} / 0302080]$;

X. Zhang, Mod. Phys. Lett. A 20, 2575 (2005) [astro-ph/0503072]

M. Szydlowski, Phys. Lett. B 632, 1 (2006), [astro-ph/0502034];

M. Szydlowski, A. Kurek, and A. Krawiec Phys. Lett. B642, 171, (2006) [astro$\mathrm{ph} / 0604327]$;

M. Szydlowski,T. Stachowiak, R. Wojtak, Phys. Rev. D 73, 063516, ,(2006);

M. R. Setare, Phys. Lett. B642, 1, (2006).

[19] C. Deffayet, G. R. Dvali and G. Gabadadze, Phys. Rev. D 65, 044023 (2002) [astro$\mathrm{ph} / 0105068]$

V. Sahni and Y. Shtanov, JCAP 0311, 014 (2003) [astro-ph/0202346];

M. R. Setare, Phys. Lett. B642, 421, (2006).

[20] A. G. Cohen, D. B. Kaplan and A. E. Nelson, Phys. Rev. Lett. 82, 4971 (1999) [hep-th/9803132];

P. Horava and D. Minic, Phys. Rev. Lett. 85, 1610 (2000) [hep-th/0001145];

S. D. Thomas, Phys. Rev. Lett. 89, 081301 (2002);

S. D. H. Hsu, Phys. Lett. B 594, 13 (2004) [hep-th/0403052]; 
K. Enqvist and M. S. Sloth, Phys. Rev. Lett. 93, 221302 (2004) [hep-th/0406019];

D. Pavon and W. Zimdahl, Phys. Lett. B 628, 206 (2005) [gr-qc/0505020];

E. Elizalde, S. Nojiri, S. D. Odintsov and P. Wang, Phys. Rev. D 71, 103504 (2005) [hep-th/0502082];

B. Hu and Y. Ling, Phys. Rev. D 73, 123510 (2006) [hep-th/0601093];

M. R. Setare, Phys. Lett. B 644, 99, (2007);

M. R. Setare, 01, 023, JCAP (2007);

X. Zhang, Phys. Rev. D 74, 103505 (2006) [astro-ph/0609699];

M. R. Setare, J. Zhang, and X. Zhang, JCAP 0703, 007, (2007);

M. R. Setare, Phys. Lett. B648, 329, (2007).

[21] A. Yu. Kamenshchik, U. Moschella, and V. Pasquier, Phys. Lett. B511, 265, (2001).

[22] D. Bazeia, R. Jackiw, Ann. Phys. 270 (1998) 246; D. Bazeia, Phys. Rev. D 59 (1999) 085007; R. Jackiw, A.P. Polychronakos, Commun. Math. Phys. 207 (1999) 107; N. Ogawa, Phys. Rev. D62, 085023, (2000); M. Szydlowski, W. Czaja, Phys. Rev. D 69, 023506, (2004).

[23] N. Bilic, G.B. Tupper and R.D. Viollier, Phys. Lett. B535 (2002) 17; N. Bilic, G.B. Tupper and R.D. Vio- llier, astro-ph/0207423.

[24] M. Bordemann and J. Hoppe, Phys. Lett. B317 (1993) 315; J.C. Fabris, S.V.B. Gonsalves and P.E. de Souza, Gen. Rel. Grav. 34 (2002) 53.

[25] M.C. Bento, O. Bertolami and A.A. Sen, Phys. Lett. B575 (2003) 172; A. A. Sen, and Robert J. Scherrer, Phys. Rev. D 72, 063511, (2005).

[26] H. Kim, H. W. Lee and Y. S. Myung, Phys. Lett. B 632, 605, (2006).

[27] C. L. Bennett et al., Astrophys. J. Suppl. 148, 1 (2003); D. N. Spergel, Astrophys. J. Suppl. 148, 175, (2003).

[28] M. Tegmark et al., Phys. Rev. D 69, 103501, (2004).

[29] B. Wang, Y. Gong, and E. Abdalla, Phys. Lett. B 624, 141 (2005).

[30] V. Gorini, A. Kamenshchik, U. Moschella and V. Pasquier, gr-qc/0403062.

[31] M. R. Setare, Eur. Phys. J. C50, 991, (2007).

[32] W. Zimdahl, 0705.2131 [gr-qc]. 